\title{
$\begin{array}{lllllll}\mathbf{J} & \mathbf{o} & \mathbf{u} & \mathbf{r} & \mathbf{n} & \mathbf{a} & \mathbf{l}\end{array}$ \\ Nonlinear Science
}

ERRATUM

\section{Erratum to: Breathers for the Discrete Nonlinear Schrödinger Equation with Nonlinear Hopping}

\author{
N.I. Karachalios • B. Sánchez-Rey • \\ P.G. Kevrekidis · J. Cuevas
}

Published online: 16 April 2013

(C) Springer Science+Business Media New York 2013

Erratum to: J Nonlinear Sci (2013) 23:205-239

DOI 10.1007/s00332-012-9149-y

The authors would like to add the following line to the Acknowledgements section:

The research of B.S.R. was funded by MICINN project FIS2008-02873.

The online version of the original article can be found under doi:10.1007/s00332-012-9149-y.

N.I. Karachalios $(\bowtie)$

Department of Mathematics, University of the Aegean, Karlovassi, 83200 Samos, Greece

e-mail: karan@aegean.gr

B. Sánchez-Rey · J. Cuevas

Grupo de Física No Lineal, Departamento de Física Aplicada I, Universidad de Sevilla,

C/Virgen de Africa, 7, 41011 Sevilla, Spain

P.G. Kevrekidis

Department of Mathematics and Statistics, University of Massachusetts,

Lederle Graduate Research Tower, Amherst, MA 01003-9305, USA 